\title{
Bioanalytical outsourcing strategy in China
}

\author{
"As a major driver in the emerging market, bioanalytical outsourcing \\ in China has evolved into a significant global player in the past \\ decade..."
}

First draft submitted: 2 June 2017; Accepted for publication: 7 June 2017;

Published online: 1 August 2017

Keywords: bioanalytical outsourcing $\bullet$ China $\bullet$ strategy

Improving $R \& D$ efficiency has been a focus of the Pharmaceutical industry over the past few decades. Externalization, such as outsourcing, is one of the strategic approaches that could give resource flexibility and increase cost-effectiveness. Since bioanalysis is an integral component of the preclinical and clinical evaluations of drug candidates, a well thought bioanalytical outsourcing strategy could lay a good foundation for future success.

\section{Industry trends in bioanalytical outsourcing}

The Pharmaceutical industry undergoes paradigm changes in portfolio focus. Introduction of new modalities, such as drug antibody-protein conjugates, oligonucleotides and cell and gene therapies, inevitably results in changes in scientific expertise extension and resource allocation. In addition, the emphasis on early engagement of translational science, such as biomarker analysis, to increase confidence in target engagement opens a new arena for bioanalytical scientists. To embrace these new challenges, bioanalytical outsourcing becomes an effective option, which offers flexibility and extension of the resources [1]. Moreover, many $\mathrm{CROs}$ have established strong infrastructure, scientific expertise quality of standards and have also become experienced in regulatory inspections.

\section{China is a global player in bioanalytical outsourcing}

With 30 years of rapid social and economic growth, China has become the second larg- est world economy. Great market potential and large patient population present unique attractiveness for investment. The Chinese government has also invested heavily in the healthcare sector, with the aim of promoting innovation, drug quality and accessibility.

From a practical perspective, Chinese regulators often require Phase I and Phase III trials in the Chinese population to demonstrate safety and efficiency of the medicine, which have already been approved for foreign countries. Additionally, due to the active promotion of innovative drug discovery and popular local generic Pharma businesses, bioanalytical support has inevitably become a high-demand business in China.

Although some Pharma companies have in-house bioanalysis capabilities for discovery or other exploratory analysis, the majority of them (especially domestic companies) do not have regulated bioanalysis capability. Conversely, it has been appreciated that scientific expertise in bioanalysis has grown rapidly in China in the past decade with an influx of western trained returnees as well as locally fostered talents who have driven the local capabilities to the global standards in scientific expertise, quality system and infrastructure.

\section{Current status of bioanalysis in China}

The bioanalytical capabilities, from both regulatory and technical perspectives, have been growing rapidly in China in the past decade. The local players feature CROs,
Kelly Dong ${ }^{1} \&$ Daniel Tang ${ }^{2}$

'GlaxoSmithKline R\&D China, Shanghai, China

${ }^{2}$ United-Power PharmaTech, Beijing,

China

Author for correspondence:

dtang69@yahoo.com 
academic and hospital-affiliated laboratories. Besides regular CFDA inspections, several global CROs and local research institutes have successfully passed foreign regulatory inspections from the US FDA, OECD, WHO and MHRA. In the meantime, many laboratories have established strong technical capabilities, including small molecule, biologics and sometimes biomarker analysis using variety of bioanalytical techniques such as LC-MS/MS and immunoassay for drug concentration and immunogenicity assessments.

It is also noteworthy that the quality standards among bioanalytical suppliers are still largely variable. In order to raise the quality of standards and enforce regulatory compliance, the CFDA announced the self inspection requirement for 1622 Bioequivalence ANDA submissions in July 2015. Sponsors could withdraw the submission if any data integrity and regulatory compliance issues are identified during self inspection. Comprehensive regulatory inspections to both clinical sites and bioanalytical laboratories are then conducted for all the studies remaining in the submission [2]. Although such activities, particularly the inspection standards, have led to significant impact on the local generic industry, clinical study sites as well as bioanalytical laboratories, they are generally welcomed as a positive drive to enhance overall clinical study quality in China. It can not only benefit the local patient population, but also lay a solid stepping stone for the organic growth of the Chinese Pharma industry on the global stage.

\section{"Western Pharma companies often choose preferred vendors, most of whom are CROs with strong technical expertise, capability and high- quality systems."}

Despite the rapid growth of bioanalytical capabilities in China and their roles in global preclinical/clinical drug development, the local bioanalytical society still faces many challenges compared with their counterparts globally.

- First, clinical sample import and export remains a specific challenge in China. Sample import permits must be in place for any sample shipments from foreign countries. A study-related sample import permit should be applied at least 2 months in advance. Clinical sample export is generally recognized as the biggest obstacle for global multicenter studies, owing to the tight control of human genetic information. We do not recommend sample export as the first choice because the success of obtaining such permit may not be guaranteed;
- Second, infectious sample handling, such as those from HIV patients, presents a challenge for many bioanalytical laboratories, both operationally and ethically;

- Third, the requirement for method cross-validation in support of global studies will inevitably increase the set-up cost, the risk of failure and possible delay in method validation completion timeline [3];

- Fourth, specific local regulatory requirements may increase the management effort. For example, the Chinese version of study reports and 100\% chromatograms (particularly bioequivalence studies) are required for studies supporting of CFDA submission [4];

- Last, the dynamic local job market often leads to a relative high staff turnover rate and increases operational cost.

Considering all these challenges, it becomes particularly important to establish a robust bioanalytical outsourcing strategy in China, to warrant the future success.

\section{Bioanalytical outsourcing strategies in China} Scope of outsourcing activities

Three types of bioanalytical outsourcing activities are involved in China:

- Bioanalysis in drug discovery is relatively straightforward, which is often handled as part of DMPK, PKPD or non-GLP toxicity evaluations. Several large CROs in China have become major global discovery partners, for multinational Pharma companies;

- Bioanalysis in GLP preclinical safety studies, which can be managed by the affiliated GLP-accredited bioanalytical laboratories or independent bioanalytical laboratories;

- Bioanalysis for clinical studies (BE for generic drugs, drug registration or part of global clinical studies for novel drugs), which are conducted in clinical centers or independent bioanalytical laboratories.

\section{Choose the right partner}

Western Pharma companies often choose preferred vendors, most of whom are CROs with strong technical expertise, capability and high-quality systems. However, many of these western CROs may not have the full presence in China or their operations may be more clinically focused. In addition, the experience in 
dealing with sample handling and regulatory requirements may be largely variable among bioanalytical suppliers. Therefore, the selection of bioanalytical partner should be in line with the study objectives rather than simple extension of the existing western partners.

For China-specific studies (IND or clinical registration studies submitted to CFDA), a high-quality local bioanalytical CRO might often provide better services due to its deep understanding of local regulatory requirements and close connection to the regulatory agency. For global multicenter clinical studies whose data will be submitted to the FDA or European Medicines Agency, it would be ideal to analyze all clinical samples at a single CRO (global or domestic laboratories) with its quality system aligned with global standards and with sufficient capability and capacity. Such selection could avoid future challenges in data comparability, the increased effort in study management and cost with different CROs.

We want to emphasize that nowadays, the selection of a bioanalytical outsourcing partner should be a business-driven decision rather than a cost-saving-only exercise. Unlike 10 years ago when cost saving was the most attractive feature to use Chinese CROs, the labor costs have been increasing dramatically in recent years, along with improving technical capability and regulatory compliance of the service providers. We suggest the selection criteria should be primarily based on capability and quality, and then throughput and cost. It is important for the sponsors to establish small numbers of strategic preferred partners, which could be different from the ones used in the USA or EU.

\section{Best practice in external bioanalytical study management}

The bioanalytical study monitor should have sound bioanalytical background and good knowledge of regulation, which allow them to promptly identify issues and provide effective guidance for troubleshooting during the course of the study. Seamless communication, periodical site visit and QA inspection could provide additional assurance of study quality.

For global multicenter studies and Phase III studies, it is important to establish such points of contact between the CRO and sponsors to minimize the potential risk of accidental disclosure of blinded information to the clinical team. The required language for local regulatory submission is language proficiency in Chinese, which would be beneficial to ensure seamless communication and study report quality. Considering the relative high turnover rate, it is useful to generate a best practice guide to lay out the detailed requirements. It is also worth noting that, unlike in western countries, sponsor support during regulatory inspection is often required in China, particularly for studies involved in different parties for sample analysis, pharmacokinetic and statistical analysis. As a result, sponsors should be prepared for this activity.

\section{Conclusion}

As a major driver in the emerging market, bioanalytical outsourcing in China has evolved into a significant global player in the past decade with a variety of bioanalytical outsourcing options. An effective bioanalytical outsourcing strategy and facilitating its execution are important to ensure the success of local drug innovation for product registration as well as contributing to global clinical development.

\section{Financial \& competing interests disclosure}

The authors have no relevant affiliations or financial involvement with any organization or entity with a financial interest in or financial conflict with the subject matter or materials discussed in the manuscript. This includes employment, consultancies, honoraria, stock ownership or options, expert testimony, grants or patents received or pending, or royalties.

No writing assistance was utilized in the production of this manuscript.

\section{References}

1 Summerfield S, Evans C, Spooner N, Dunn J, Szapacs M, Yang E. Integrating internal and external bioanalytical support to deliver a diversified pharmaceutical portfolio. Bioanalysis 6(10), 1311-1319 (2014).

2 Zhong D, Dong K, Wei M, Li J, Tang D. Update on Chinese Regulated Bioanalysis. Bioanalysis Zone (2016). www.bioanalysis-zone.com/2016/02/07/update

Georgiou A, Dong K, Hughes S, Barfield M. An interlaboratory transfer of a multi-analyte assay between continents. Bioanalysis 7(7), 825-831 (2015).

4 The Guideline for Method Validation of Quantitative Analysis of Biological Samples. Chinese Pharmacopoeia (2015). www.edqm.eu/sites/default/files/1710_1130_brief 